\title{
New Design Methodology for Impact Angle Control Guidance for Various Missile and Target Motions
}

\author{
Min-Guk Seo, Chang-Hun Lee and Min-Jea Tahk
}

\begin{abstract}
This paper introduces a new design methodology for impact angle control guidance (IACG) laws. The proposed methodology can extend any proven homing guidance laws to their impact angle control versions if the expressions of the estimated terminal flight path angles under those guidance laws are given. The time derivatives of the estimated terminal flight path angles are obtained as functions of the guidance commands. The IACG versions of the homing guidance laws are derived from those functions and the desired error dynamics of the estimated terminal flight path angle. The guidance law of each IACG version has two terms: The first term maintains the characteristics and capturability of the original guidance law and the second term drives the estimated terminal flight path angle to converge to the specified flight path angle. When a well-understood homing guidance law for a certain combination of target and missile models is given, an IACG law for that combination is easily derived without reformulating the guidance problem again. The usefulness of the proposed method is demonstrated by several examples, deriving new IACG laws for various target and missile models.
\end{abstract}

Index Terms - Missile Guidance, Impact Angle Control Guidance, Velocity-varying Missile, Maneuvering Target, Accelerated Target

\section{INTRODUCTION}

$\mathrm{A}$ NTI-TANK missiles are most effective when the terminal impact angles are orthogonal to the target armor. Anti-ship missiles must control their terminal impact angles to avoid ship defense systems, like CIWS (close-in weapon system). The infrared seekers of anti-aircraft missiles are effective when tracking the jet flame of a target at its rear.

To attack these various types of targets efficiently, impact angle control guidance (IACG) laws have been developed over several decades to address these unique conditions. The IACG laws for stationary targets have been detailed in previous studies [1-10]. Various control and guidance theories, like

This work was conducted at High-Speed Vehicle Research Center of KAIST with the support of the Defense Acquisition Program Administration and Agency for Defense Development. (Contract number: UD170018CD)

M.-G. Seo is with the Department of Aerospace Engineering, KAIST, Daejeon 305-701, Korea (e-mail: mgseo@fdcl.kaist.ac.kr).

C.-H. Lee is with Cranfield University, College Road, Cranfield MK43 0AL, Bedfordshire, United Kingdom (e-mail: lckdgns@gmail.com)

M.-J. Tahk is with the Department of Aerospace Engineering, KAIST, Daejeon 305-701, Korea (e-mail: mjtahk@fdcl.kaist.ac.kr). optimal control [1-3,6,7], proportional navigation guidance (PNG) law [4] and its biased form [5,10], time-to-go polynomial guidance [8], and sliding mode control [9], have been applied to solve the problem. Moving targets with constant velocity are handled by synthesizing PNG algorithm [11] and applying the time-varying biased PNG algorithm [12]. The IACG algorithms in [13-20] deal with maneuvering targets. Nonlinear control theories, such as backstepping control [13], optimal control [14], differential game theory [14], and sliding mode control [15-20], are utilized in those references. A general form of IACG law is devised in [21]. This form is based on the optimal control theories and handles first-order lag systems. However, when defining the general dynamics model in [21], targets are assumed to be stationary and the velocity of missile is constant. The meaning of 'general' in [21] is that the proposed IACG law design methodology deals with the minimization of control effort weighted by a generalized timeto-go function.

In these previous studies, the authors applied the following design strategy when developing their own IACG algorithms. First, the engagement dynamics for a certain class of target and missile motions under consideration is set up. Then, the traditional homing guidance problem is reformulated in conjunction with additional terminal constraints on the impact angle, considering the engagement dynamics.

Accordingly, this design approach has some fundamental drawbacks. First, the IACG law obtained by this approach is only applicable to the certain class of target and missile motions under consideration. Hence, if we want to extend the capability of an already designed IACG law to a different class of target and missile motions, it must be reformulated with different engagement dynamics. Second, even in the case where a user has a capturability-proven homing guidance law for a certain set of missile and target motions, the user has to reformulate guidance law design problem with a terminal impact angle constraint and solve it to get IACG law. Generally, the linkage between the pre-existing homing guidance law and the newly designed IACG law diminishes in this kind of approaches. Therefore, the obtained IACG law can also be regarded as a totally new one, which departs from the pre-existing homing guidance law. This requires additional efforts to fully understand the characteristics of the obtained IACG law, even though there is a continuation of the guidance characteristics.

The IACG law design approach of this paper is motivated by 
the impact time control guidance law design technique proposed by [22], which explicitly uses the time-to-go prediction formula to derive a class of impact time control guidance law. In this paper, we apply this technique for impact angle control by deriving the expressions of the terminal impact angles for various target and missile models.

The proposed IACG law consists of two terms: The first term is for maintaining the characteristics of the original guidance law and guaranteeing capturability. The second term is for impact angle control, which diminishes to zero at the terminal time so as not to interfere with the capturability of the original guidance law. In the proposed design approach, any existing homing guidance laws for various kinds of target motions can still be utilized. Also, the proposed design methodology is able to consider various missile motions during the design step, constant velocity as well as varying velocity, when the missile velocity profile is given.

Consider the advantages of the IACG law design methodology of this paper, which simply derives a new IACG law from a given homing guidance law. First, the obtained IACG law could directly inherit the characteristics of the original guidance law. Also, since the IACG laws converge to their original versions as the terminal impact angle error diminishes to zero, the continuities between the two versions are remained. If characteristics of the homing guidance law are known, there would be no need to devote extra effort to understand those of the obtained IACG law. Second, a large number of existing homing guidance laws could be used to devise IACG laws for various target and missile motions.

In order to demonstrate these characteristics, in this paper, several IACG laws are extended from homing guidance laws for various target motions, including stationary targets, constant turn-rate targets and constant axial-acceleration targets. Numerical simulations are conducted to show the characteristics and the performances of the devised IACG laws.

This paper is organized as follows: Section II introduces the relative dynamics between missile and target. The IACG law design methodology is addressed in Section III and the IACG law design samples are given in Section IV. Simulation results showing the validity of the sample guidance laws are presented in Section IV. The concluding remarks are given in Section V.

\section{SYSTEM MODELING}

This section describes the derivation of engagement dynamics between missile and target. As shown in Fig. 1, two frames are utilized in this study: $\left(O_{I}, X_{I}, Y_{I}\right)$ is the inertial frame, and $\left(O_{R}, X_{R}, Y_{R}\right)$ is the reference frame obtained by rotating $\left(O_{I}, X_{I}, Y_{I}\right)$ by angle $\theta$ with its origin at the initial target position. The purpose of introducing the reference frame is to linearize the nonlinear engagement dynamics in this frame.

In Fig. 1, the missile and target are denoted by $M$ and $T$, respectively. The relative distance and the line-of-sight (LOS) angle between them are represented by $R$ and $\sigma$, respectively. The notations $V_{M}, \gamma_{M}, A_{M}$, and $u_{M}$ represent the velocity, the flight path angle, the axial acceleration, and the

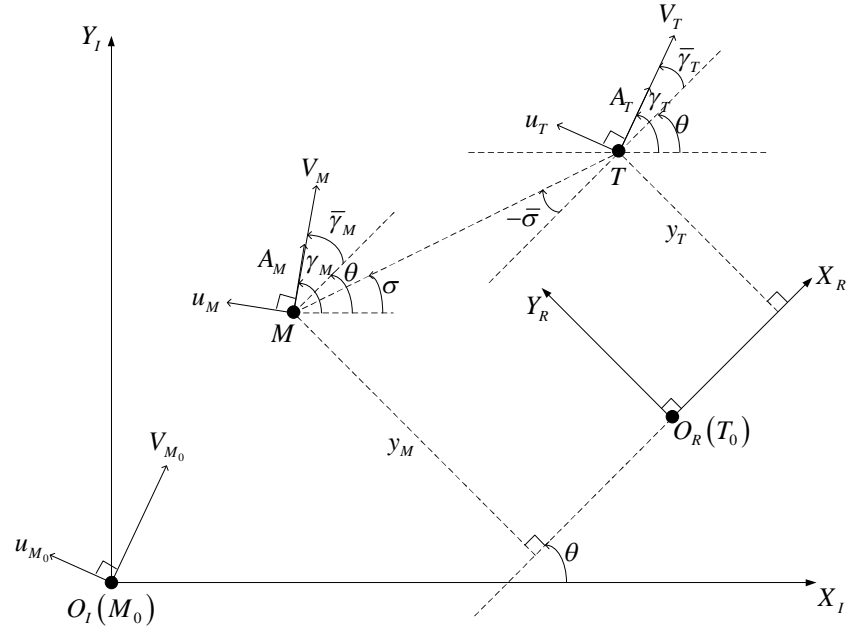

Fig. 1. Engagement Geometry

maneuver acceleration of the missile, respectively. In the missile system, $u_{M}$ is the control input which changes $\gamma_{M} \cdot V_{T}$, $\gamma_{T}, A_{T}$, and $u_{T}$ are defined for the target in a similar way. Additionally, in $\left(O_{R}, X_{R}, Y_{R}\right)$, the angles are defined as,

$$
\begin{gathered}
\bar{\gamma}_{(\cdot)}=\gamma_{(\cdot)}-\theta \\
\bar{\sigma}=\sigma-\theta
\end{gathered}
$$

(.) is a subscript; it becomes $M$ when a variable or parameter is related to missile, and it is substituted by $T$ for target cases. Then, the system dynamics are expressed in $\left(O_{R}, X_{R}, Y_{R}\right)$.

$$
\begin{aligned}
& \dot{y}_{(\cdot)}=V_{(\cdot)} \sin \bar{\gamma}_{(\cdot)} \\
& \dot{v}_{(\cdot)}=u_{(\cdot)} \cos \bar{\gamma}_{(\cdot)}+A_{(\cdot)} \sin \bar{\gamma}_{(\cdot)} \\
& \dot{V}_{(\cdot)}=A_{(\cdot)}
\end{aligned}
$$

where $y_{(\cdot)}$ and $v_{(\cdot)}$ are the lateral position and the velocity of the missile or target along the $Y_{R}$-axis.

From Eqs.(2.a)-(2.c), we can obtain the linearized engagement dynamics when the reference frame with $\theta$ is defined to make $\bar{\gamma}_{M}, \bar{\gamma}_{T}$, and $\bar{\sigma}$ small enough.

$$
\begin{aligned}
& \dot{y}_{(\cdot)}=V_{(\cdot)} \bar{\gamma}_{(\cdot)} \triangleq v_{(\cdot)} \\
& \dot{v}_{(\cdot)}=u_{(\cdot)}+A_{(\cdot)} \bar{\gamma}_{(\cdot)} \\
& \dot{V}_{(\cdot)}=A_{(\cdot)}
\end{aligned}
$$

The angle $\theta$ is a design parameter, which is chosen by users to simplify and linearize the engagement dynamics into a simple form. $\theta$ is usually chosen as the line-of-sight angle at the beginning of the homing phase. In most cases, the engagement geometry at the beginning of the terminal homing phase is controlled during the midcourse guidance phase to enhance the capturability of the missile. Also, the terminal homing phases are usually short. Those statements imply that the trajectory changes of missile and target are small during the terminal homing phase, and the missile and target are expected to gradually get closer in this phase. Thus, it is assumed that users can choose a proper $\theta$ for small angle approximation. Those small angle approximations and $\theta$ are usually applied in the 
previous missile guidance law studies. Moreover, although the IACG design methodology proposed in this paper is based on those approximations, the simulation results in this paper show that the guidance laws derived with this methodology are applicable for nonlinear engagement cases. In the linearized engagement dynamics, $\bar{\sigma}$ is approximated as below:

$$
\bar{\sigma} \simeq \frac{\left(y_{T}-y_{M}\right)}{R}
$$

From the dynamics in Eqs.(3.a)-(3.c), the interception condition is given by

$$
y_{T_{f}}-y_{M_{f}}=0
$$

where $y_{T_{f}}$ and $y_{M_{f}}$ are the target and missile lateral positions at the final time $t_{f}$, respectively. Since the missile approaches target during terminal homing phase, $t_{f}$ is defined as the time when $x_{T}=x_{M}$. For the impact angle control problem, the aspect angle $\Gamma$ is another important parameter to be controlled, which is defined as $\Gamma \triangleq \gamma_{T}-\gamma_{M}$. If a desired $\Gamma$ is given as $\Gamma_{d}$, the condition of impact angle control can be written as,

$$
\Gamma_{d}=\gamma_{T_{f}}-\gamma_{M_{f}}
$$

where $\gamma_{T_{f}}$ and $\gamma_{M_{f}}$ are the final values of the target and missile flight path angles, respectively.

\section{IACG LAW DESIGN METHOdOLOGY}

In this section, we introduce the design methodology for impact angle control guidance. In the proposed method, the existing guidance laws for the interception can be simply extended to their impact angle control versions using the expressions of the estimated terminal flight path angles.

In order to do that, we first derive the general expression of the estimated terminal flight path angle when a capturability -proven missile guidance command for a certain class of target motion (i.e., $u_{C}$ ) is applied to the missile systems, such as $u_{M}=u_{C}$. Then, in that case, the estimated value of lateral velocity at the terminal time (i.e., $\hat{v}_{M_{f}}$ ) is obtained from the dynamics given in Eq.(3.b).

$$
\hat{v}_{M_{f}}=v_{M}(t)+\int_{t}^{t_{f}}\left[u_{C}(\tau)+A_{M}(\tau) \bar{\gamma}_{M}(\tau)\right] d \tau
$$

Eqs.(1.a) and (3.a) result in the following relationship.

$$
\hat{v}_{M_{f}}=\hat{V}_{M_{f}}\left(\hat{\gamma}_{M_{f}}-\theta\right)
$$

where $\hat{V}_{M_{f}}$ represents the estimated terminal missile velocity, which is given from Eq.(3.c).

$$
\hat{V}_{M_{f}}=V_{M}(t)+\int_{t}^{t_{f}} A_{M}(\tau) d \tau
$$

Then, by combining Eqs.(7) and (8), we can determine the estimated terminal flight path angle, $\hat{\gamma}_{M_{f}}$.

$$
\hat{\gamma}_{M_{f}}=\frac{1}{\hat{V}_{M_{f}}}\left[v_{M}(t)+\eta\right]+\theta
$$

where

$$
\eta=\int_{t}^{t_{f}}\left[u_{C}(\tau)+A_{M}(\tau) \bar{\gamma}_{M}(\tau)\right] d \tau
$$

Note that $\hat{\gamma}_{M_{f}}$ is given by the function of $u_{C}$ and $v_{M}$. Most capturability-proven missile guidance commands are generally given by functions of the relative dynamics information, such as $\sigma$ and $R$. For some cases, the target information is also used for the guidance command, if the target information is available by means of estimation. Also, from Eq.(3.a), we can observe that $v_{(\cdot)}$ is given as the function of $V_{(\cdot)}$ and $\gamma_{(\cdot)}$, respectively. Therefore, in general, $\hat{\gamma}_{M_{f}}$ can be expressed by the functions of all those state variables, as discussed above.

$$
\hat{\gamma}_{M_{f}}=f\left(\sigma, R, \gamma_{M}, V_{M}, \gamma_{T}, V_{T}\right)
$$

Accordingly, we can generally express the time derivative of $\hat{\gamma}_{M_{f}}$ using the partial derivatives of those parameters.

$\dot{\hat{\gamma}}_{M_{f}}=\frac{\partial f}{\partial \sigma} \dot{\sigma}+\frac{\partial f}{\partial R} \dot{R}+\frac{\partial f}{\partial \gamma_{M}} \dot{\gamma}_{M}+\frac{\partial f}{\partial V_{M}} \dot{V}_{M}+\frac{\partial f}{\partial \gamma_{T}} \dot{\gamma}_{T}+\frac{\partial f}{\partial V_{T}} \dot{V}_{T}$

Among the terms in Eq.(13), the time derivative of $\gamma_{M}$ is given by $\dot{\gamma}_{M}=u_{M} / V_{M}$ from the dynamics relation. Then, applying this relation to Eq.(13) and rearranging the result, we have

$$
\dot{\hat{\gamma}}_{M_{f}}=\alpha+\beta u_{M}
$$

where

$$
\begin{gathered}
\alpha=\frac{\partial f}{\partial \sigma} \dot{\sigma}+\frac{\partial f}{\partial R} \dot{R}+\frac{\partial f}{\partial V_{M}} \dot{V}_{M}+\frac{\partial f}{\partial \gamma_{T}} \dot{\gamma}_{T}+\frac{\partial f}{\partial V_{T}} \dot{V}_{T} \\
\beta=\frac{1}{V_{M}} \frac{\partial f}{\partial \gamma_{M}}
\end{gathered}
$$

Note that the above equation provides the direct relationship between the control input $u_{M}$ and the time derivative of $\hat{\gamma}_{M_{f}}$. This result implies that $\hat{\gamma}_{M_{f}}$ can be controlled by imposing $u_{M}$ when $\partial f / \partial \gamma_{M} \neq 0$. This fact is the key point in developing the proposed design methodology.

Hereafter, the proposed guidance command $u_{M}$, which drives $\hat{\gamma}_{M_{f}}$ to a desired value $\gamma_{d}$, will be determined. For convenience of the derivation, we assume that the proposed guidance command $u_{M}$ consists of two terms, as below:

$$
u_{M}=u_{0}+u_{1}
$$

where $u_{0}$ is defined as the term which maintains the change of $\hat{\gamma}_{M_{f}}$ as zero (i.e., $\dot{\hat{\gamma}}_{M_{f}}=0$ ). In other words, $\hat{\gamma}_{M_{f}}$ is not altered by $u_{0}$. And, $u_{1}$ is defined as an additional command term to achieve the desired value of $\hat{\gamma}_{M_{f}}$. By definition, substituting $u_{0}$ into Eq.(14) gives

$$
\alpha+\beta u_{0}=0
$$

Then, $u_{0}$ is obtained from Eq.(18).

$$
u_{0}=-\frac{\alpha}{\beta}
$$

Interestingly, as proven in Lemma 1 , the obtained guidance command $u_{0}$ becomes the original guidance law $u_{C}$. Therefore, we can predict that $u_{0}$ retains the characteristics and 
capturability of the original guidance law.

Lemma 1. The guidance command $u_{0}$ as given in Eq.(19) is identical to the original guidance law $u_{C}$.

Proof) From Eq.(10), $\dot{\gamma}_{M_{f}}$ is determined as follows:

$$
\dot{\hat{\gamma}}_{M_{f}}=\frac{1}{\hat{V}_{M_{f}}}\left[\dot{v}_{M}(t)+\dot{\eta}\right]
$$

The following equations are obtained from Eq.(3.b) and Eq.(11).

$$
\begin{gathered}
\dot{v}_{M}(t)=u_{M}(t)+A_{M}(t) \bar{\gamma}_{M}(t) \\
\dot{\eta}=-\left[u_{C}(t)+A_{M}(t) \bar{\gamma}_{M}(t)\right]
\end{gathered}
$$

$\dot{\hat{\gamma}}_{M_{f}}$ is rewritten by substituting Eqs.(21) and (22) into Eq.(20).

$$
\dot{\hat{\gamma}}_{M_{f}}=\frac{1}{\hat{V}_{M_{f}}}\left[u_{M}(t)-u_{C}(t)\right]
$$

$u_{M}=u_{0}$ is the guidance command to maintain $\dot{\hat{\gamma}}_{M_{f}}=0$ by definition. Therefore, imposing this condition on Eq.(23) gives

$$
u_{0}(t)=u_{C}(t)
$$

which completes the proof.

Next, $u_{1}$ is designed to accomplish $\gamma_{d}$. In order for that to occur, let the impact angle error $\varepsilon_{\gamma}$ be first defined.

$$
\varepsilon_{\gamma}=\gamma_{d}-\hat{\gamma}_{M_{f}}
$$

We choose the desired error dynamics of $\varepsilon_{\gamma}$ as follows:

$$
\dot{\varepsilon}_{\gamma}+k\left(\frac{\varepsilon_{\gamma}}{t_{g o}}\right)=0, \text { for } k>0
$$

where $k$ is the design parameter. $t_{g o}$ is the time-to-go, which is defined as,

$$
t_{g o} \triangleq t_{f}-t
$$

In order to achieve $\gamma_{d}$ at $t_{f}, \varepsilon_{\gamma}$ should converge to zero during the interception. The finite time convergence of $\varepsilon_{\gamma}$ can be proven in Lemma 2.

Lemma 2. Under the error dynamics shown in Eq.(26), the impact angle error $\varepsilon_{\gamma}$ goes to zero as $t_{g o} \rightarrow 0$.

Proof) The differential equation given in Eq.(26) is a sort of Cauchy-Euler equation. It can be converted into an ordinary differential equation by introducing a new independent variable $\zeta$, which is defined as below:

$$
t_{g o}=e^{\zeta}
$$

Eq.(26) is rewritten by applying Eq.(28).

$$
\frac{d \varepsilon_{\gamma}}{d \zeta}-k \varepsilon_{\gamma}=0
$$

The solution of Eq.(29) is obtained as,

$$
\varepsilon_{\gamma}=K e^{k \zeta}
$$

where $K$ is a constant. By substituting Eq.(28) into Eq.(30), the following equation is derived.

$$
\varepsilon_{\gamma}=K t_{g o}^{k}
$$

$$
K=\left[\frac{\varepsilon_{\gamma_{0}}}{t_{f}^{k}}\right]
$$

where $\varepsilon_{\gamma_{0}}$ is the initial value of $\varepsilon_{\gamma}$ and $t_{f}$ denotes the terminal time. $\varepsilon_{\gamma}$ is obtained from Eq.(31) and Eq.(32).

$$
\varepsilon_{\gamma}=\left[\frac{\varepsilon_{\gamma_{0}}}{t_{f}^{k}}\right] t_{g o}^{k}
$$

It is observed from Eq.(33) that $\varepsilon_{\gamma}$ converges to zero as $t_{g o} \rightarrow 0$ in $k>0$ cases, which completes the proof.

The main reason to introduce this error dynamics is to drive $\varepsilon_{\gamma}$ to zero within a finite $t_{f}$. Also, since the trend of $\varepsilon_{\gamma}$ with this error dynamics becomes Eq.(33), which is a $t_{g o}$ polynomial form, the users are able to easily predict the shape of $\varepsilon_{\gamma}$ decrement. Due to those two advantages, the desired error dynamics in Eq.(26) is utilized in this paper. This error dynamics is also utilized in [23], which deals with the impact time and angle control guidance law design.

Then, $u_{1}$ is derived from Eqs.(14), (17), (19) and (26) under the assumption that $\gamma_{d}$ is constant.

$$
u_{1}=\frac{k}{\beta}\left(\frac{\varepsilon_{\gamma}}{t_{g o}}\right)
$$

Finally, the IACG law denoted by $u_{M}$ can be obtained by combining Eqs.(19) and (34).

$$
u_{M}=-\frac{\alpha}{\beta}+\frac{k}{\beta}\left(\frac{\gamma_{d}-\hat{\gamma}_{M_{f}}}{t_{g o}}\right)
$$

Note that the second term of Eq.(35) converges to zero at the terminal time as proven in Lemma 2. Therefore, we can infer that the IACG command converges to $u_{0}$ as $t_{g o} \rightarrow 0$ and that it possess the same characteristics as $u_{C}$, which means the capturability of $u_{M}$ is guaranteed.

Also, it is shown from Eqs.(9) and (10) that $\hat{\gamma}_{M_{f}} \rightarrow \gamma_{M_{f}}$ as $t \rightarrow t_{f}$. This can be rewritten from Eq.(27) that $\hat{\gamma}_{M_{f}} \rightarrow \gamma_{M_{f}}$ as $t_{g o} \rightarrow 0$, meaning that $\hat{\gamma}_{M_{f}}$ becomes more accurate as the missile approaches the target. Since Lemma 2 shows that $\hat{\gamma}_{M_{f}} \rightarrow \gamma_{d}$ as $t_{g o} \rightarrow 0$, from these observations, we can finally predict that $\gamma_{M_{f}} \rightarrow \gamma_{d}$ as $t_{g o} \rightarrow 0$.

The above result shows that the design methodology of this paper is able to generate a IACG law with just the $\hat{\gamma}_{M_{f}}$ of a capturability-proven guidance law. Namely, if the expressions of $\hat{\gamma}_{M_{f}}$ are known for any capturability-proven guidance laws for various missile and target motions, they can be simply extended to their impact angle control versions using Eqs.(15), (16) and (35). Therefore, if we already have capturabilityproven guidance laws, we do not need to reformulate the guidance problem and start all over again to obtain IACG laws.

$K$ is obtained from the initial value of $\varepsilon_{\gamma}$ and Eq.(31). 


\section{EXAMPLES OF IACG LAW DESIGN}

In this section, some sample cases will be provided to help readers better understand how our design methodology works. In order to demonstrate the effectiveness of the proposed design methodology, we consider caputrability-proven guidance laws for various missile and target motions, as discussed below.

\section{A. Constant Speed Missile \& Stationary Target}

First, we consider a constant speed missile and a stationary target. It is well-known that the pure proportional navigation (PPN) guidance law is effective for this missile and target motion. PPN guidance command is given by

$$
u_{C}=N V_{M} \dot{\sigma}
$$

where $N$ is the navigation constant. According to [4], it is already known that $\hat{\gamma}_{M_{f}}$ for PPN is obtained as,

$$
\hat{\gamma}_{M_{f}}=\frac{N}{N-1} \sigma-\frac{1}{N-1} \gamma_{M}
$$

Then, $\alpha$ and $\beta$ are calculated by applying Eqs.(15) and (16) to Eq.(37).

$$
\begin{gathered}
\alpha=\frac{N}{N-1} \dot{\sigma} \\
\beta=-\frac{1}{V_{M}(N-1)}
\end{gathered}
$$

Finally, the impact angle control version of PPN is simply determined by substituting Eqs.(38) and (39) into Eq.(35).

$$
u_{M}=N V_{M} \dot{\sigma}-\frac{k V_{M}(N-1)}{t_{g o}}\left(\gamma_{d}-\hat{\gamma}_{M_{f}}\right)
$$

Note that the first term in Eq.(40) is identical to the original homing guidance law in Eq.(36) and this result matches with Lemma 1. Also, in the case of $k=1$, Eq.(40) is equal to the IACG law as obtained in [10]. If $N=n+2$ and $k=m+1$ for $n>m \geq 0$ are chosen, then the above guidance command can be converted to TPG (time-to-go polynomial guidance) as detailed in [8]. Therefore, we can predict that the proposed method is a more general approach to designing IACG laws.

\section{B. Constant Missile Axial-Acceleration}

\section{\& Constant Target Turn-Rate}

In this example, the target is assumed to perform maneuvers with a constant turn-rate (i.e., $\dot{u}_{T}=0$ ) and the missile is assumed to have a constant axial acceleration (i.e., $\dot{A}_{M}=0$ ). For this target and missile motion, a capturability-proven homing guidance law $u_{C}$ is given as,

$$
u_{C}=N V_{M_{\text {avg }}} \dot{\sigma}+\frac{N^{\prime}}{2} u_{T}-A_{M} \bar{\gamma}_{M}
$$

where

$$
N^{\prime}=\frac{V_{M_{\text {avg }}}}{V_{C_{\text {avg }}}} N
$$

The proof of the capturability of the guidance law in Eq.(41) is given in Appendix. It also contains the definitions of $V_{M_{\text {avg }}}$ and
$V_{C_{a v g}}$ in Eq.(42). In this case, $\hat{\gamma}_{M_{f}}$ is given by (see Appendix)

$$
\begin{aligned}
\hat{\gamma}_{M_{f}}=\frac{1}{\hat{V}_{M_{f}}}\left[\frac{N V_{M_{\text {avg }}}}{N^{\prime}-1} \sigma-\frac{V_{M}}{N^{\prime}-1} \gamma_{M}+\frac{N^{\prime} V_{T}}{N^{\prime}-1} \bar{\gamma}_{T}\right. \\
\left.+\left(\frac{V_{M}-N V_{M_{\text {avg }}}}{N^{\prime}-1}+\hat{V}_{M_{f}}\right) \theta+\frac{N^{\prime} u_{T}}{2\left(N^{\prime}-1\right) V_{C_{\text {avg }}}} R\right]
\end{aligned}
$$

Then, $\alpha$ and $\beta$ are calculated from Eqs.(15), (16) and (43) with small angle approximation, $\cos \bar{\gamma}_{T} \simeq 1$.

$$
\begin{gathered}
\alpha=\frac{N}{N^{\prime}-1} \frac{V_{M_{\text {avg }}}}{\hat{V}_{M_{f}}} \dot{\sigma}+\frac{N^{\prime}}{2\left(N^{\prime}-1\right)} \frac{1}{\hat{V}_{M_{f}}} u_{T}-\frac{1}{N^{\prime}-1} \frac{1}{\hat{V}_{M_{f}}} A_{M} \bar{\gamma}_{M} \\
\beta=-\frac{1}{N^{\prime}-1} \frac{1}{\hat{V}_{M_{f}}}
\end{gathered}
$$

In a similar way, the impact angle control version of $u_{C}$ as shown in Eq.(41) is derived as,

$u_{M}=N V_{M_{\text {avg }}} \dot{\sigma}+\frac{N^{\prime}}{2} u_{T}-A_{M} \bar{\gamma}_{M}-k \frac{\hat{V}_{M_{f}}\left(N^{\prime}-1\right)}{t_{g o}}\left(\gamma_{d}-\hat{\gamma}_{M_{f}}\right)$

For a target with the constant turn rate, $\gamma_{d}$ is given as below:

$$
\gamma_{d}=\gamma_{T}+\frac{u_{T}}{V_{T}} t_{g o}+\Gamma_{d}
$$

Note that the first to third terms in Eq.(46) are identical to the original homing guidance law as we predicted from Lemma 1.

\section{Constant Missile Axial-Acceleration \\ \& Constant Target Axial-Acceleration}

Next, both the target and missile are assumed to be boosted with a constant axial-acceleration (i.e., $\dot{A}_{T}=0$ and $\dot{A}_{M}=0$ ). To consider those target and missile motions, a $u_{C}$ that guarantees capturability is given as,

$$
u_{C}=N V_{M_{\text {avg }}} \dot{\sigma}+\frac{N^{*}}{2} A_{T} \bar{\gamma}_{T}-A_{M} \bar{\gamma}_{M}
$$

where

$$
\begin{aligned}
& N^{*}=\frac{V_{M_{\text {avg }}}}{V_{C_{\text {avg }}}} N \\
& V_{M_{\text {avg }}}=V_{M}+\frac{A_{M}}{2} \frac{R}{V_{C_{\text {avg }}}} \\
& \hat{V}_{M_{f}}=V_{M}+A_{M} \frac{R}{V_{C_{\text {avg }}}} \\
& V_{C_{\text {avg }}}=\frac{1}{2}\left(V_{C}+\sqrt{V_{C}^{2}+2\left[A_{M} \cos \left(\gamma_{M}-\sigma\right)-A_{T} \cos \left(\gamma_{T}-\sigma\right)\right] R}\right) \\
& V_{C}=V_{M} \cos \left(\gamma_{M}-\sigma\right)-V_{T} \cos \left(\gamma_{T}-\sigma\right)
\end{aligned}
$$

The capturability of the guidance law in Eq.(48) is able to be verified as the way similar to Appendix. The IACG law for the constant $A_{M}$ and $A_{T}$ case is determined from the algorithm proposed in Section III and Eq.(48). 
$u_{M}=N V_{M_{a r g}} \dot{\sigma}+\frac{N^{*}}{2} A_{T} \bar{\gamma}_{T}-A_{M} \bar{\gamma}_{M}-k \frac{\hat{V}_{M_{f}}\left(N^{*}-1\right)}{t_{g o}}\left(\gamma_{d}-\hat{\gamma}_{M_{f}}\right)$

where

$$
\begin{aligned}
& \gamma_{d}=\gamma_{T}+\Gamma_{d} \\
& \hat{\gamma}_{M_{f}}=\frac{1}{\hat{V}_{M_{f}}}\left[\frac{N V_{M_{\text {avg }}}}{N^{*}-1} \sigma-\frac{V_{M}}{N^{*}-1} \gamma_{M}+\frac{N^{*} V_{T}}{N^{*}-1} \bar{\gamma}_{T}\right. \\
& \left.+\left(\frac{V_{M}-N V_{M_{\text {arg }}}}{N^{*}-1}+\hat{V}_{M_{f}}\right) \theta+\frac{N^{*} A_{T}}{2\left(N^{*}-1\right) V_{C_{\text {arg }}}} R \bar{\gamma}_{T}\right]
\end{aligned}
$$

The obtained guidance law in Eq.(54) contains its original homing guidance command (i.e., the first to third terms).

\section{Implementation Issues}

In order to implement the guidance laws derived with the proposed design methodology, the obtained guidance commands basically require information about the $R, \sigma, \dot{\sigma}$, $V_{M}, \gamma_{M}, A_{M}, V_{T}, \gamma_{T}, A_{T}$, or $u_{T}$. In general, those parameters can be measured or estimated using seeker and inertial navigation systems (INS).

Also, IACG laws designed with the proposed methodology require $t_{g o}$, as shown in Eq.(35). For practicality, the following $t_{g o}$ estimation method is used in this study.

$$
t_{g o}=\frac{R}{V_{C_{\text {avg }}}}
$$

Note that, although the linearized engagement dynamics model in Eqs.(3.a)-(3.c) is utilized during the derivations of the guidance laws in this section, the linearized terms are substituted with the nonlinear variables, like $\sigma$ or $\gamma_{M}$, in the final guidance law expressions in Eqs.(40), (46), and (54). This implies that the sample IACG laws are applicable for the nonlinear guidance geometries.

\section{NUMERICAL RESULTS}

Simulations were conducted to examine the performances and characteristics of the sample IACG laws for two scenarios:

Scenario A - Constant $A_{M}$ Missile \& Constant $u_{T}$ Target

Scenario B - Constant $A_{M}$ Missile \& Constant $A_{T}$ Target The parameter settings for the simulations are addressed in Table 1. For each scenario, five different $\gamma_{d}$ are defined. The guidance law in Eq.(46) is applied for Scenario A, and Scenario B is conducted with the law in Eq.(54). All the simulations are performed with the nonlinear dynamics model in Eqs.(2.a) $-(2 . c)$.

Fig. 2 and 3 show the simulation results of Scenarios A and B. The results without the impact angle control terms $(k=0)$ are also addressed. Scenarios A and B result in a significant missile velocity drop as shown in Fig. 2 (b) and Fig. 3 (b). Considering the worst case in each scenario, Case 1 of Scenario A shows about $37 \%$ missile velocity drop in 15 seconds while the target is performing $3 g$ of maneuver, and Case 1 of Scenario B shows about 55\% missile velocity drop and almost 167\% target velocity increase in about 6.7 seconds. Moreover, since the simulations are conducted with nonlinear dynamics model, the small angle approximations are broken during the simulations. Fig. 2 (d) and Fig. 3 (e) show that, although engagement conditions are dramatically varying with time and small angle approximations are invalid, the IACG law for each scenario achieves the given terminal impact angle constraints.

The initial value of $u_{0}$ is the same for all the cases of a certain scenario, as given in Eq.(19). The remaining part of the guidance command, $u_{1}$, is defined in Eq.(34) and it is proportional to $\varepsilon_{\gamma}$. Since the initial value of $u_{1}$ is proportional to the initial $\varepsilon_{\gamma}$ as shown in Eq.(34), for the same scenario, the differences on the initial guidance command between $k=0$ case and other cases are proportional to the initial $\varepsilon_{\gamma}$. Those are observed in Fig. 2 (c) and Fig. 3 (d).

\section{CONCLUSION}

A new methodology to design IACG laws is proposed in this paper. The proposed methodology enables existing homing guidance laws to be extended to IACG laws, while maintaining their original characteristics. Any kind of homing guidance law can be extended to IACG laws for various target motions using the proposed methodology, with only its estimated terminal flight path angle equation. In addition, cases of varying missile velocity can be accommodated if their profiles are known.

The guidance law designed by the proposed methodology is composed of two terms: one for the objectives of the original guidance law and the other for impact angle control. As examples, guidance law designs for a stationary target, and for constant axial acceleration missiles against constant turn-rate or constant axial-acceleration targets were addressed, and the guidance performances were demonstrated using computer simulations.

Based on the proposed methodology, IACG laws for various target motions and missile velocity profiles will be developed in future studies.

\section{APPENDIX}

This appendix provides the proof of the capturability of the homing guidance law shown in Eq.(41). The capturability will be proven by showing that the lateral positions of missile and target at the terminal time are identical. First, $R$ is approximated as $t_{g o}$ multiplied by the average closing velocity of missile and target in the time interval $\left[\begin{array}{ll}t & t_{f}\end{array}\right], V_{C_{\text {arg }}}$.

$$
R \simeq V_{C_{\text {avg }}} t_{g o}
$$

The derivation of $V_{C_{\arg }}$ starts from the closing velocity at time $t, V_{C}(t)$, which is given by

$$
V_{C}(t)=V_{M} \cos \left(\gamma_{M}(t)-\sigma(t)\right)-V_{T} \cos \left(\gamma_{T}(t)-\sigma(t)\right)
$$

Under the assumption that the variations on $\left(\gamma_{M}-\sigma\right)$ and 
$\left(\gamma_{T}-\sigma\right)$ in $\left[\begin{array}{ll}t & t_{f}\end{array}\right]$ are small, the time derivative of $V_{C}$ is given as the following constant value in this time interval.

$$
\dot{V}_{C}=A_{M} \cos \left(\gamma_{M}(t)-\sigma(t)\right)
$$

Eq.(60) results in the relationship between $V_{C}(t)$ and $V_{C_{f}}$ as,

$$
V_{C_{f}}^{2}-V_{C}(t)^{2}=2 A_{M} \cos \left(\gamma_{M}(t)-\sigma(t)\right) R(t)
$$

where $V_{C_{f}}$ represents the closing velocity at the terminal time. Then, from Eq.(61), $V_{C_{\text {avg }}}$ in $\left[\begin{array}{ll}t & t_{f}\end{array}\right]$ is determined as below:

$$
V_{C_{\text {avg }}}=\frac{1}{2}\left[V_{C}(t)+\sqrt{V_{C}^{2}(t)+2 A_{M} \cos \left(\gamma_{M}(t)-\sigma(t)\right) R(t)}\right]
$$

and $V_{C_{\text {avg }}}$ is set to be constant in $\left[\begin{array}{ll}t & t_{f}\end{array}\right] . \dot{R}$ is obtained from Eq.(58).

$$
\dot{R}=-V_{C_{\text {avg }}}
$$

The average value and estimated terminal value of missile velocity, $V_{M_{\text {avg }}}$ and $\hat{V}_{M_{f}}$, are given from Eqs.(3.c) and (58).

$$
\begin{gathered}
V_{M_{\text {avg }}}=V_{M}+\frac{A_{M}}{2} \frac{R}{V_{C_{\text {avg }}}} \\
\hat{V}_{M_{f}}=V_{M}+A_{M} \frac{R}{V_{C_{\text {avg }}}}
\end{gathered}
$$

$\dot{\sigma}$ is obtained from Eqs.(1.b), (3.a), (4), and (58).

$$
\dot{\sigma}=\frac{1}{V_{C_{\text {avg }}} t_{\text {go }}}\left(v_{T}-v_{M}\right)+\frac{1}{V_{C_{\text {avg }}} t_{\text {go }}^{2}}\left(y_{T}-y_{M}\right)
$$

The following differential equation is given from Eqs.(3.a), (3.b), (41), and (66).

$$
\begin{aligned}
& \ddot{y}_{M}+N^{\prime} \frac{1}{\tau_{g o}} \dot{y}_{M}+N^{\prime} \frac{1}{\tau_{g o}^{2}} y_{M} \\
& =N^{\prime} \frac{1}{\tau_{g o}^{2}}\left(y_{T}(t)+v_{T}(t) t_{g o}+\frac{1}{2} u_{T} t_{g o}^{2}\right)
\end{aligned}
$$

where

$$
\tau_{g o}=t_{f}-\tau \quad \tau \in\left[\begin{array}{ll}
t & t_{f}
\end{array}\right]
$$

Since the above equation is given as a well-known CauchyEuler equation form, the solution of Eq.(67) is simply obtained.

$$
\begin{gathered}
y_{M}=C_{1} \tau_{g o}^{N^{\prime}}+C_{2} \tau_{g o}+y_{T}(t)+v_{T}(t) t_{g o}+\frac{1}{2} u_{T} t_{g o}^{2} \\
v_{M}=-N^{\prime} C_{1} \tau_{g o}^{N^{\prime}-1}-C_{2}
\end{gathered}
$$

where

$$
\begin{gathered}
C_{1}=-\frac{1}{N^{\prime}-1} \frac{1}{t_{g o}^{N^{\prime}}}\left[-\left(y_{T}(t)+v_{T}(t) t_{g o}+\frac{1}{2} u_{T} t_{g o}^{2}\right)\right. \\
\left.+v_{M}(t) t_{g o}+y_{M}(t)\right] \\
C_{2}=\frac{1}{N^{\prime}-1} \frac{1}{t_{g o}}\left[-N^{\prime}\left(y_{T}(t)+v_{T}(t) t_{g o}+\frac{1}{2} u_{T} t_{g o}^{2}\right)\right. \\
\left.+v_{M}(t) t_{g o}+N^{\prime} y_{M}(t)\right]
\end{gathered}
$$

The terminal value of lateral position, $y_{M_{f}}$, is obtained by applying $\tau=t_{f}$ to Eq.(69).

$$
y_{M_{f}}=y_{M}\left(t_{f}\right)=y_{T}(t)+v_{T}(t) t_{g o}+\frac{1}{2} u_{T} t_{g o}^{2}=y_{T_{f}}
$$

which completes the capturability proof of $u_{C}$ in Eq.(41).

From Eqs.(1.a), (1.b), (3.a), (4) and (69), $v_{M_{f}}$ of the guidance law in Eq.(41) is obtained as follows:

$$
\begin{aligned}
v_{M_{f}}= & \frac{N V_{M_{\text {avg }}}}{N^{\prime}-1} \sigma-\frac{V_{M}}{N^{\prime}-1} \gamma_{M}+\frac{N^{\prime} V_{T}}{N^{\prime}-1} \bar{\gamma}_{T}+\left(\frac{V_{M}-N V_{M_{\text {avg }}}}{N^{\prime}-1}\right) \theta \\
& +\frac{N^{\prime}}{2\left(N^{\prime}-1\right)} \frac{u_{T}}{V_{C_{\text {avg }}}} R
\end{aligned}
$$

Finally, the expression of $\hat{\gamma}_{M_{f}}$ shown in Eq.(43) is obtained by applying the relationship in Eq.(8).

\section{REFERENCES}

[1] Song, T. L., Shin, S. J., and Cho, H., "Impact Angle Control for Planar Engagements," IEEE Transactions on Aerospace and Electronic Systems, Vol. 35, No. 4, 1999, pp. 1439-1444.

[2] Ryoo, C. K., Cho, H., and Tahk, M. J., "Optimal Guidance Laws with Terminal Impact Angle Constraints," Journal of Guidance, Control, and Dynamics, Vol. 28, No. 4, 2005, pp. 724-732.

[3] Ryoo, C. K., Cho, H., and Tahk, M. J., "Time-to-Go Weighted Optimal Guidance with Impact Angle Constraints," IEEE Transactions on Control Systems Technology, Vol. 14, No. 3, 2006, pp. 483-492.

[4] Ratnoo, A., and Ghose, D., "Impact Angle Constrained Interception of Stationary Targets," Journal of Guidance, Control, and Dynamics, Vol. 31, No. 6, 2008, pp. 1816-1821.

[5] Erer, K. S., and Merttopcuoglu, O., "Indirect Impact-angle-control Against Stationary Targets using Biased Pure Proportional Navigation," Journal of Guidance, Control, and Dynamics, No. 35, Vol. 2, 2012, pp. 700-704.

[6] Lee, Y. I., Kim, S. H., Lee, J. I., and Tahk, M. J., "Analytic solutions of generalized impact-angle-control guidance law for firstorder lag system," Journal of Guidance, Control, and Dynamics, Vol. 36, No. 1, 2012, pp. 96-112.

[7] Lee, Y. I., Kim, S. H., and Tahk, M. J., "Optimality of Linear Time Varying Guidance for Impact Angle Control," IEEE Transactions on Aerospace and Electronic Systems, Vol. 48, No. 4, 2012 pp. 2802-2817.

[8] Lee, C.-H., Kim, T.-H., Tahk, M.-J., and Whang, I.-H., "Polynomial Guidance Laws Considering Terminal Impact Angle and Acceleration Constraints," IEEE Transactions on Aerospace and Electronic Systems, Vol. 49, No. 1, 2013, pp. 74-92.

[9] Lee, C.-H., Kim, T.-H., and Tahk, M.-J., "Design of Impact Angle Control Guidance Laws via High-performance Sliding Mode Control," Proceedings of the Institution of Mechanical Engineers, Part G: Journal of Aerospace Engineering, Vol. 227, No. 2, 2013, pp.235-253.

[10] Lee, C.-H., Kim, T.-H., and Tahk, M.-J., "Intercept Angle Control Guidance Using Proportional Navigation with Error Feedback," Journal of Guidance, Control, and Dynamics, Vol. 36, No. 5, Sep.-Oct. 2013, pp. $1556-1561$.

[11] Ratnoo, A., and Ghose, D., "Impact Angle Constrained Guidance against Nonstationary Nonmaneuvering Targets," Journal of Guidance, Control, and Dynamics, Vol. 33, No. 1, Jan. 2010, pp. 269-275.

[12] Kim, B. S., Lee, J. G., and Han, H. S., "Biased PNG Law for Impact with Angular Constraint," IEEE Transactions on Aerospace and Electronic Systems, Vol. 34, No. 1, 1998, pp. 277-288.

[13] Kim, K., Jung, B., and Kim, Y., "Practical Guidance Law Controlling Impact Angle," Proceedings of the Institution of Mechanical Engineers, Part G: Journal of Aerospace Engineering, Vol. 221, No. 1, 2007, pp. 2936.

[14] Shaferman, V., and Shima, T., "Linear Quadratic Guidance Laws for Imposing a Terminal Intercept Angle," Journal of Guidance, Control, and Dynamics, Vol. 31, No. 5, 2008, pp. 1400-1412.

[15] Shima, T., "Intercept-angle Guidance," Journal of Guidance, Control, and Dynamics, Vol. 34, No. 2, 2011, pp. 484-492.

[16] Kumar, S. R., Rao, S., and Ghose, D., "Sliding-mode Guidance and Control for All-aspect Interceptors with Terminal Angle Constraints," 
Journal of Guidance, Control, and Dynamics, Vol. 35, No. 4, 2012, pp 1230-1246.

[17] Zhang, Z., Li, S., and Luo, S., "Terminal Guidance Laws of Missiles Based on ISMC and NDOB with Impact Angel Constraint," Aerospace Science and Technology, Vol. 31, No. 1, 2013, pp. 30-41.

[18] Kumar, S. R., Rao, S., and Ghose, D., "Nonsingular Terminal Sliding Mode Guidance with Impact Angle Constraints," Journal of Guidance, Control, and Dynamics, Vol. 37, No. 4, 2014, pp.1114-1130.

[19] He, S., Lin, D., and Wang, J., "Continuous Second-order Sliding Mode Based Impact Angel Guidance Law," Aerospace Science and Technology, Vol. 41, 2015, pp. 199-208.

[20] Cho, D., Kim, H. J., and Tahk, M.-J., "Impact Angle Constrained Sliding Mode Guidance Against Maneuvering Target with Unknown
Acceleration," IEEE Transactions on Aerospace and Electronic Systems, Vol. 51, No. 2, 2015, pp. 1310-1323.

[21] Lee, Y. I., Kim, S. H., Lee, J. I., and Tahk, M. J., "Analytic Solutions of Generalized Impact-Angle-Control Guidance Law for Firstorder Lag System," Journal of Guidance, Control, and Dynamics, Vol. 31, No. 1, 2012, pp. 96-112.

[22] Tahk, M. J., Shim, S. W., Hong, S. M., and Lee, C. H., "Impact Time Control Based on Time-to-go Prediction for Sea-skimming Anti-ship Missiles," Submitted for publication to IEEE Transactions on Aerospace and Electronic Systems, July 2016.

[23] Harl, N., and Balakrishnan, S. N., "Impact Time and Angle Guidance With Sliding Mode Control," IEEE Transactions on Control Systems Technology, Vol. 20, No. 6, 2012, pp. 1436-1449.

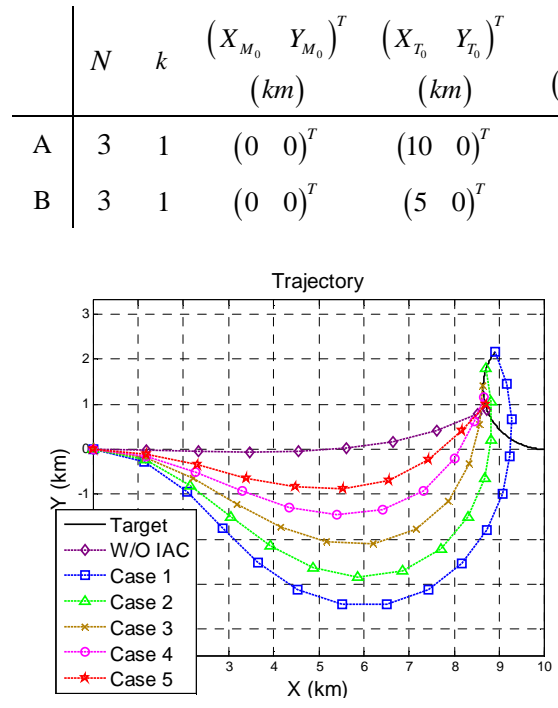

Fig. 2 (a) Trajectory

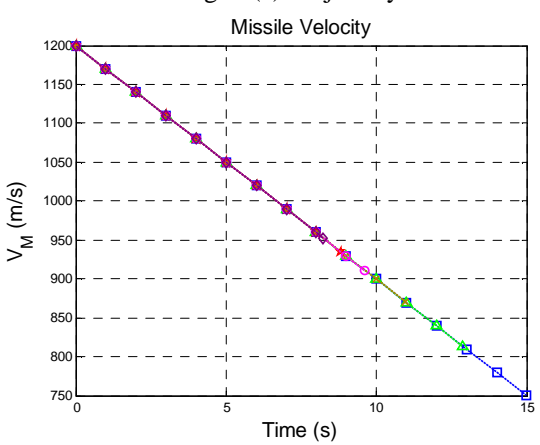

Fig. 2 (b) Missile Velocity

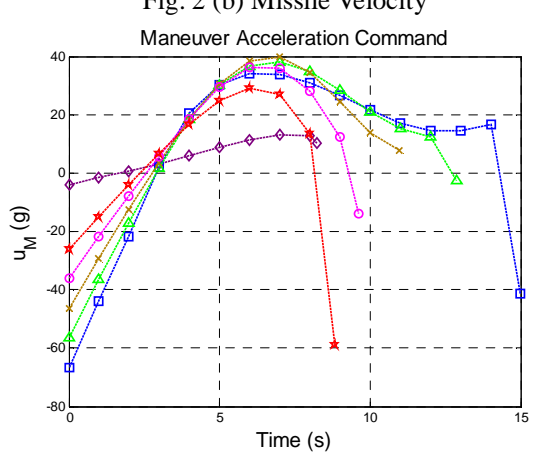

Fig. 2 (c) Maneuver Acceleration Command Fig. 2. Results of Scenario A

Table 1 Simulation Parameters

\begin{tabular}{|c|c|c|c|c|c|c|c|c|c|c|c|}
\hline $\begin{array}{c}V_{M} \\
(m / s)\end{array}$ & $\begin{array}{l}\gamma_{M_{0}} \\
\left({ }^{\circ}\right)\end{array}$ & $\begin{array}{c}A_{M} \\
\left(m / s^{2}\right)\end{array}$ & $\begin{array}{c}V_{T_{0}} \\
(m / s)\end{array}$ & $\begin{array}{l}\gamma_{T_{0}} \\
\left({ }^{\circ}\right)\end{array}$ & $\begin{array}{c}u_{T} \\
(g)\end{array}$ & $\begin{array}{c}A_{T} \\
\left(m / s^{2}\right)\end{array}$ & Case 1 & Case 2 & $\begin{array}{l}\Gamma_{d}\left({ }^{\circ}\right) \\
\text { Case } 3\end{array}$ & Case 4 & Case 5 \\
\hline 1200 & 0 & -30 & 200 & 180 & -3 & 0 & -60 & -30 & 0 & 30 & 60 \\
\hline 1200 & 0 & -100 & 100 & 135 & 0 & 25 & 0 & 45 & 90 & 135 & 180 \\
\hline
\end{tabular}

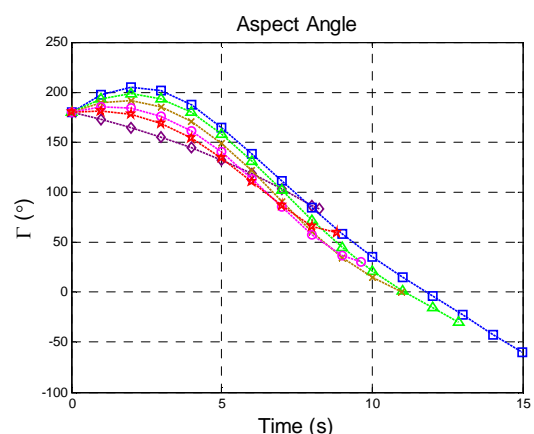

Fig. 2 (d) Aspect Angle

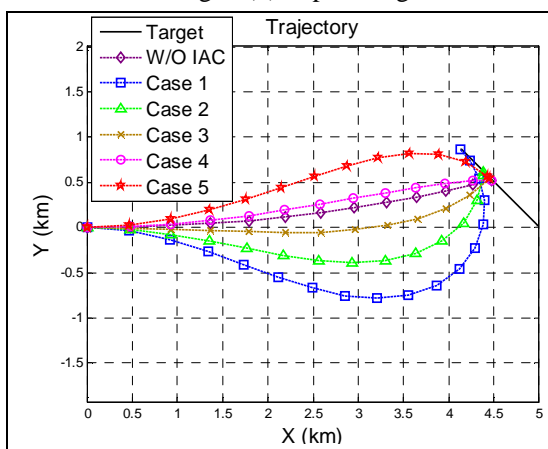

Fig. 3 (a) Trajectory Missile Velocity

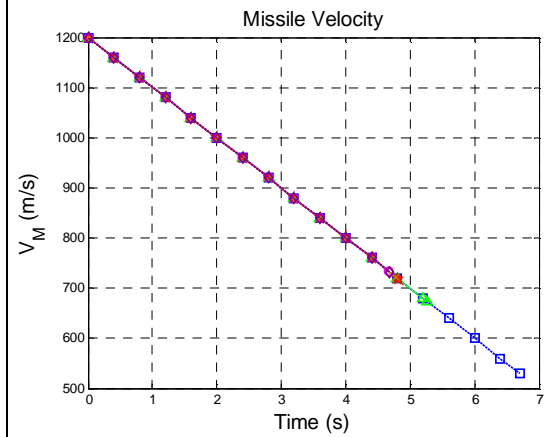

Fig. 3 (b) Missile Velocity

Fig. 3. Results of Scenario B

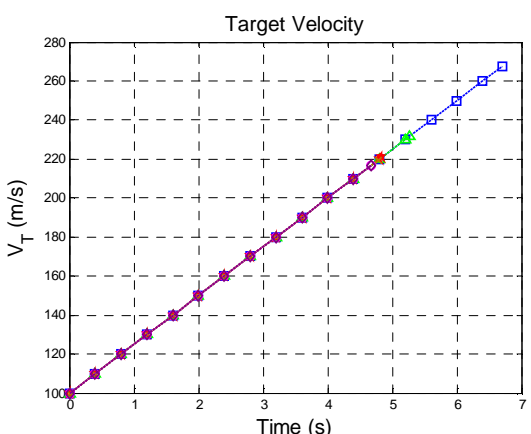

Fig. 3 (c) Target Velocity

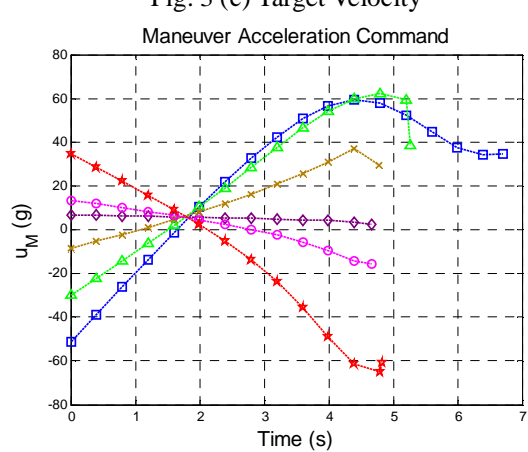

Fig. 3 (d) Maneuver Acceleration Command

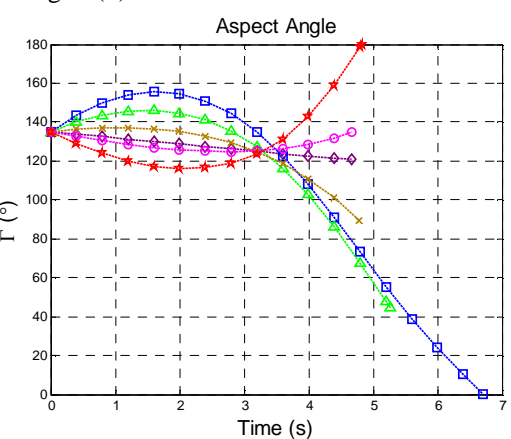

Fig. 3 (e) Aspect Angle 
2017-09-26

New design methodology for impact angle control guidance for various missile and target motions

Seo, Min-Guk

IEEE

Seo MG, Lee CH, Tahk MJ. (2018) New design methodology for impact angle control guidance for various missile and target motions. IEEE Transactions on Control Systems Technology, Volume 26, Issue 6, November 2018, pp. 2190-2197

http://dx.doi.org/10.1109/TCST.2017.2749560

Downloaded from Cranfield Library Services E-Repository 